\title{
Frecuencia de diagnósticos y tratamientos pulpares según indicadores de la demanda realizados en una clínica dental universitaria
}

Gonzales A, Quintana M, Matta C, Maldonado M. Frecuencia de diagnósticos y tratamientos pulpares según indicadores de la demanda realizados en una clínica dental universitaria. Rev Estomatol Herediana 2005; 15 (2) : 150 - 154.

\section{RESUMEN}

Los diagnósticos y tratamientos pulpares que fueron realizados en los pacientes del pregrado de la Clínica Estomatológica Central de la Facultad de Estomatología Roberto Beltrán Neira de la Universidad Peruana Cayetano Heredia durante el período 2003 al 2004, se relacionaron según los indicadores de la demanda. Se encontró que la mayor frecuencia de tratamientos pulpares fueron las pulpectomías anteriores $(50,2 \%)$, seguidas de las pulpectomías en molares $(24,8 \%)$ y por ultimo las pulpectomías en premolares $(24,7 \%)$. Los diagnósticos pulpares mas frecuentemente hallados correspondieron a pulpitis aguda $(45,1 \%)$, pulpa vital $(27,4 \%)$, encontrándose mayor tratamiento en el sexo femenino.

Palabras clave: NECESIDADES Y DEMANDA DE SERVICIOS DE SALUD. estadística \& datos numéricos / PULPA DENTARIA. patología.

Frequency of pulpar diagnostics and treatment by demand indicators in a college dental clinic

ABSTRACT

Pulpar diagnoses and treatments carried out in undergraduate patients at the Peruvian University Cayetano Heredia Roberto Beltrán Neira Stomathology School during 2003 to 2004 period, were related to demand indicators. Anterior pulpectomies were found to have the highest prevalence of pulpar treatment $(50.2 \%)$, followed by molar pulpectomies $(24.8 \%)$ and premolar pulpectomies $(24.7 \%)$. The most frequently pulpar diagnoses was acute pulpitis $(45.1 \%)$, vital pulp $(27.4 \%)$. The majority of the patients were women.

Keywords: HEALTH SERVICES NEEDS AND DEMAND. statistics \& numerical data / DENTAL PULP. pathology

\section{Alan Gonzales Román ${ }^{1}$ Martín Quintana del Solar ${ }^{2}$ Carlos Matta Morales ${ }^{2}$ María de las Mercedes Maldonado Mendoza ${ }^{2}$}

\section{${ }^{1}$ Cirujano Dentista}

${ }^{2}$ Docente del Departamento Académico de Clínica Estomatológica. Facultad de

Estomatología. Universidad Peruana Cayetano Heredia.

\section{Correspondencia}

Martín Quintana del Solar

Av. Honorio Delgado 430 Lima - 31, Perú

Teléfono: (51-1) 481-3380

e-mail: martin34@ec-red.com

\section{Introducción}

El profesional deberá constantemente decidir si la pulpa ha sido dañada en forma reversible o irreversible, para así poder brindarle un tratamiento adecuado al paciente. El diagnóstico correcto es el procedimiento de aceptar un paciente, reconocer que tiene un problema, determinar la causa de este problema y desarrollar un plan de tratamiento que ayude a resolverlo, brindándole atención en salud dental. Para evaluar la calidad de la atención en salud se utilizan las variables del contexto (normas vigentes), demanda (estadística de los pacientes), oferta (estructura hospitalaria), procesos (administrativos y atención médica), resultados (producción del hospital) e impacto (costo-efecto de la atención). Los indicadores de la demanda son edad, sexo, grado de instrucción, procedencia, ocupación y mes de atención del paciente, los que determinarán tanto el tipo como la necesidad de tratamiento (1-15).
El conocimiento del estado de la pulpa dentaria es imprescindible para elaborar y plantear un adecuado tratamiento. En la endodoncia este procedimiento es crucial por lo que exige todo nuestro esfuerzo y destreza, considerándose que estamos decidiendo sobre la vida o muerte de un tejido tan noble como es el tejido pulpar (16-20).

Estudios determinaron que el mayor número de tratamientos de conductos se ubicó a los 36 años, no hallando diferencia significativa entre las edades según el genero, también se planteó que las mujeres reciben terapia de canales con mayor frecuencia, debido a que disponen de mas tiempo para el tratamiento $(21,22)$. Con respecto al genero, diversos estudios hallaron que en su mayoría pertenecen al genero femenino (23-29), encontraron que la mayor cantidad de pacientes correspondían a estudiantes, amas de casa y en su mayoría eran de sexo femenino entre las edades de 20 a 35 años (30-41).
El propósito del presente estudio es determinar la frecuencia, diagnóstico y tipos de tratamientos pulpares según los indicadores de la demanda en los pacientes atendidos en Clínica Integral del Adulto de la Clínica Estomatológica Central de la Facultad de Estomatología Roberto Beltrán Neira durante los años 2003 al 2004 y así conocer la demanda de atención en tratamientos pulpares.

\section{Materiales y método}

El presente estudio fue de tipo descriptivo y corte transversal. El tipo de muestreo fue no probabilístico, tomándose todas las actividades clínicas de endondoncia, las cuales fueron 665 actividades clínicas de endodoncia de los operadores de la asignatura CIA III, IV y V, tomadas de la base de datos del registro de actividades clínicas, que se extrajeron de las mismas historias clínicas de la Clínica Estomatológica Central de la UPCH, durante el período 2003 al 2004, determinando la asociación de 
Tabla 1. Distribución de los tratamientos pulpares según la edad del paciente

\begin{tabular}{lccccccc}
\hline & \multicolumn{7}{c}{ rango de edad } \\
tratamiento & $<14$ & $14-24$ & $25-35$ & $36-46$ & $47-57$ & $>57$ & total \\
\hline pulpectomía anterior & 3 & 79 & 105 & 81 & 39 & 27 & 334 \\
pulpectomía molar & 3 & 32 & 45 & 35 & 30 & 20 & 165 \\
pulpectomía premolar & 1 & 25 & 50 & 39 & 28 & 21 & 164 \\
\hline total & 7 & 136 & 200 & 155 & 97 & 68 & 663
\end{tabular}

Tabla 2. Distribución de los tratamientos pulpares según sexo del paciente

\begin{tabular}{lccl}
\hline \multirow{2}{*}{ tratamiento } & \multicolumn{2}{c}{ sexo } & \\
\hline pulpectomía anterior & 229 & 105 & 334 \\
pulpectomía molar & 94 & 71 & 165 \\
pulpectomía premolar & 117 & 47 & 164 \\
\hline total & 440 & 223 & 663 \\
\hline
\end{tabular}

los diagnósticos y tratamientos pulpares según los indicadores de demanda que fueron: año de estudio del operador, edad, sexo, ocupación, grado de instrucción, procedencia, sector de la boca y mes de atención.

La Facultad de Estomatología Roberto Beltrán Neira (FE) de la Universidad Peruana Cayetano Heredia (UPCH) incluye asignaturas clínicas para los alumnos de pregrado en su plan curricular desde el tercer al quinto año, siendo una de ellas las Clínicas Integrales del Adulto (CIA) que van del I al V según el año en que se realicen. Las asignaturas CIA III, IV y V dentro de las actividades académicas que le corresponden, llevan el diagnóstico y tratamiento de lesiones pulpares (42).

Se realizó el análisis univariado mostrando tablas de distribución de frecuencias y porcentajes para las variables de estudio. Luego se realizó el análisis bivariado mediante tablas de doble entrada. Se estableció la asociación de las variables de estudio mediante la prueba de chi cuadrado al nivel de significancia del 0,05.

\section{Resultados}

El mayor porcentaje de pacientes atendidos oscila entre las edades de 25 y 35 años de edad $(30,1 \%)$ seguido de pacientes entre edades de 36 y 46 años $(23,3 \%)$ y en menor porcentaje son los pacientes menores de 14 años $(1,1 \%)$.
En la tabla 1, entre las edades de 25 y 35 años hay una mayor cantidad de pulpectomías anteriores (105 tratamientos) y en las edades de 14 años o menos hay una menor cantidad de pulpectomías anteriores (tres tratamientos). También entre las edades de 25 y 35 años hay mayor cantidad de pulpectomías en molares (45 tratamientos) y una menor cantidad de tratamientos entre las edades de 14 o menos (tres tratamientos). Las pulpectomías en premolar tienen mayor cantidad de tratamientos entre las edades de 25 y 35 años (50 casos), seguidas de las edades entre 36 y 46 años (39 tratamientos), siendo la menor cantidad de tratamientos entre las edades de 14 años o menos (un tratamiento).

El sexo femenino tiene mayor porcentaje de atención $(66,3 \%)$ y el sexo masculino el menor porcentaje de atención $(37,7 \%)$. El mayor porcentaje de pacientes atendidos en la clínica vienen a ser estudiantes $(27,8 \%)$, seguido de la de ama de casa $(25.9 \%)$. El mayor porcentaje de los pacientes vienen del Cono Norte (43,6\%), seguido del Área Central de Lima $(35,8 \%)$. El menor porcentaje de pacientes provienen del Cono Sur $(5,6 \%)$ y Cono Oeste $(5,6 \%)$.

En la tabla 2, para el sexo femenino se realizan más pulpectomías anteriores (229 tratamientos) y para el sexo masculino en menor cantidad (105 tratamientos). En las pulpectomías en molar también en el sexo femenino se realizan en mayor cantidad (94 tratamientos) que en los del sexo masculino (71 tratamientos). En cuanto a las pulpectomías en premolar fueron hechas en mayor cantidad en los del sexo femenino (117 tratamientos) que en los del sexo masculino (47 tratamientos).

Los tratamientos pulpares con mayor porcentaje fueron la pulpectomía anterior $(50,2 \%)$, seguida de pulpectomía en molar $(24,8 \%)$ y por ultimo el recubrimiento pulpar directo (3\%). El diagnóstico pulpar que tiene mayor porcentaje es la pulpitis aguda $(45,1 \%)$ y pulpa vital $(27,4 \%)$. El diagnostico pulpar que tiene menor porcentaje es la pulpitis reversible ( $2 \%)$.

El mayor porcentaje de donde se realizaron los tratamientos de conductos, lo tiene el sector ántero superior $(45,9 \%)$ $\mathrm{y}$ el sector con menor porcentaje es el sector ántero inferior (4,5\%). La pieza en la que se realizaron tratamientos de conductos con mayor porcentaje es la pieza $21(12,3 \%)$ seguida de la pieza 11 $(11,1 \%)$, siendo la de menor porcentaje la pieza $31(0,3 \%)$.

Las pulpectomías anteriores se realizaron en mayor cantidad en el sector ántero superior (304 tratamientos) en comparación con el sector ántero inferior (30 tratamientos). Las pulpectomías en molares se realizaron en mayor cantidad en el sector póstero inferior derecho (50 tratamientos), seguidos por el sector póstero inferior izquierdo (47 tratamientos) y en menor cantidad en el sector póstero superior izquierdo (29 tratamientos). Las pulpectomías en premolares se realizaron en mayor cantidad en el sector póstero superior derecho (50 tratamientos) y en menor cantidad en el sector póstero inferior izquierdo (32 tratamientos).

Los diagnósticos de necrosis séptica se encuentran en mayor cantidad en pacientes de 25 y 35 años (48 casos) seguidos por pacientes de 14 y 24 años (29 casos). Las pulpitis agudas se presentaron en mayor cantidad en pacientes de 25 y 35 años (86 casos), siendo en menor cantidad en pacientes menores de 14 años (3 casos). Las pulpitis crónicas se presentaron en mayor cantidad en pacientes de 36 y 46 años (9 
casos) seguidas por pacientes entre los 47 y 57 años ( 8 casos), y en menor cantidad a pacientes de 58 años a más (2 casos). Los diagnósticos de pulpa vital se presentaron en mayor cantidad en pacientes de 25 y 35 años (59 casos), seguidos por pacientes de 36 y 46 años (50 casos) y en menor cantidad en pacientes menores de 14 años (4 casos).

El diagnóstico de necrosis séptica se presentan en mayor cantidad en el sector ántero superior (62 casos) y en menor cantidad en el sector ántero inferior (3 casos). La pulpitis aguda se presenta en mayor número en el sector ántero superior (129 casos) y en menor numero en el sector ántero inferior (14 casos). La pulpitis crónica la encontramos en mayor número en el sector ántero superior (13 casos), y en menor número en el sector ántero inferior (dos casos). El diagnóstico de pulpa vital lo encontramos en mayor cantidad en el sector ántero superior (100 casos) y en menor cantidad en el sector ántero inferior (10 casos).

\section{Discusión}

Los tratamientos pulpares que obtuvieron el mayor porcentaje correspondieron a las pulpectomías anteriores $(50,2 \%)$, seguidas de las pulpectomías en molares $(24,8 \%)$ y por ultimo las pulpectomías en premolares $(24,7 \%)$. Esto se puede explicar debido a que los dientes anteriores por su morfología y ubicación son más proclives a la caries y los traumatismos en pacientes menores de 30 años según los estudios de Gulabivala (43) en 1972. Los resultados obtenidos fueron muy parecidos a los obtenidos por Barbakow (22) en 1980 donde el mayor número de dientes tratados en la región de los incisivos (22\%) fueron realizados por estudiantes de Odontología, mientras que las pulpectomías en molares (39\%) fueron realizadas con mayor frecuencia por odontólogos experimentados o especialistas en endodoncia, por ser tratamientos de mayor complejidad. Estos resultados coinciden con lo referido por Márquez (35) en 1998 quien realizó un estudio de la frecuencia de motivos y lugar de referencia para tratamiento de conductos en la Clínica del Post Grado de Endodoncia de la Facultad de Estomatología de la UPCH entre Enero de 1995 y Enero de 1997, en donde la pulpectomía en molares son los dientes más frecuentemente referidos al Post Grado (48\%) siendo referidas en menor número las pulpectomías anteriores (12\%). Ello quizá se debió a que la Clínica del Post Grado de Endodoncia esta destinada a tratar los casos complejos de endodoncia, además si se consideran los resultados obtenidos por Barbakow (22), todos los casos simples son tratados por estudiantes de odontología y los casos complejos por especialistas.

En cuanto a los diagnósticos pulpares los que obtuvieron mayor porcentaje correspondieron a pulpitis aguda $(45,1 \%)$ seguidos del diagnóstico de pulpa vital $(27,4 \%)$. Esto se explica por el alto índice de caries que afecta las piezas dentarias en la población y que ello conlleva a la posterior inflamación (pulpitis) o destrucción (necrosis) mientras que los tratamientos en pulpa viva sólo se producen en casos de iatrogénica odontológica, traumatismo o por indicaciones protésicas (44).

En cuanto a los grupos de edades, el mayor porcentaje de tratamientos pulpares se concentra principalmente en los grupos comprendidos entre 25 y 35 años de edad (30,1\%). En este sentido los resultados son parecidos a los obtenidos por Barbakow (22), que halló que la mayoría de los casos tratados endodónticamente se ubicaron en la tercera década de vida, siendo los 35 años la edad promedio. Molven (21) halló que el mayor número de tratamientos de conductos se ubicó a los 36 años dentro de su muestra. Harty (28) también halló que los 35 años es la edad promedio donde la mayoría de los pacientes reciben tratamiento endodóntico.

En relación al género, el mayor porcentaje de tratamientos de conductos lo encontramos en pacientes del sexo femenino $(66,3 \%)$ seguido del masculino $(33,7 \%)$. Esto se puede explicar por el mayor porcentaje de individuos de sexo femenino en países en vías de desarrollo y que el sexo femenino acude más a la consulta odontológica que el sexo masculino, porque solicita más tratamientos estéticos $(33,45-47)$.

En cuanto a la ocupación que tienen los pacientes que asistieron a la Clínica Estomatológica Central de la Universidad Peruana Cayetano Heredia el mayor porcentaje se concentra en los estudiantes $(27,8 \%)$, seguido de las amas de casa $(25,9 \%)$. Esto se puede deber a que los estudiantes debido a la cercanía de sus centros de estudios tienden a asistir con mayor frecuencia, ya que muchos de ellos son alumnos de la Universidad Peruana Cayetano Heredia. Además las amas de casa disponen de mayor cantidad de tiempo para asistir a la consulta odontológica y buscan tratamientos estéticos $(32,33,40)$.

En cuanto a los tratamientos pulpares según el sexo del paciente, encontramos que las pulpectomías anteriores se hallan en mayor cantidad en pacientes de sexo femenino (229 tratamientos), las pulpectomías en molar en pacientes de sexo femenino (94 tratamientos) y las pulpectomías en premolar también en pacientes de sexo femenino (117 tratamientos). Abbott (31) halló que los pacientes referidos para estos tratamientos son más del genero femenino que del masculino $(64,8 \%$ contra $35,2 \%)$. Resultados similares encontraron Manning (29) y corroborados por Harty (28). Pareciera que las mujeres reciben tratamiento endodóntico con mayor frecuencia que los varones, aunque las estadísticas pueden variar, es una tendencia confirmada por casi todos los estudios al respecto, excepto por el de Barbakow (22) que halló una ligera diferencia a favor de los casos masculinos. Molven (21) (1976), planteó un antecedente, el postuló que las mujeres reciben terapias de canales con mayor frecuencia debido a que disponen de más tiempo para el tratamiento.

\section{Conclusiones}

- Los tratamientos pulpares más realizados fueron las pulpectomías anteriores, seguidas de las pulpectomías en molares y por las pulpectomías en premolares.

- Los diagnósticos pulpares más frecuentemente hallados correspondie- 
ron a pulpitis aguda.

- En cuanto a los tratamientos pulpares según el rango de edad encontramos que la pulpectomía anterior, molar y premolar se hallan en mayor cantidad en pacientes de 25 y 35 años de edad. Respecto a los tratamientos pulpares según el sexo del paciente, encontramos que las pulpectomías anteriores, molar y se hallan en mayor cantidad en pacientes de sexo femenino.

- Con respecto a los diagnósticos pulpares según el rango de edad de los pacientes encontramos que la mayor cantidad de necrosis séptica, pulpa vital y pulpitis aguda se dan en pacientes entre 25 y 35 años de edad, y pulpitis crónica en pacientes de 36 y 46 años de edad.

\section{Referencias bibliográficas}

1. Bergenholtz G. Pathogenic mechanisms in pulpal disease. J Endod 1990; 16(2):98-101.

2. Walton R. Endodoncia principios y practica clínica. México DF: Nueva Editorial Interamericana; 1990. p.1920.

3. Navarro - Beltrán E. Diccionario Terminológico de Ciencias Médicas. 12a ed. Barcelona: Salvat; 1984. p. 360.

4. Bailit H, Braun R. Is Periodontal Disease the Primary cause of tooth extraction in adults? J Am Dent Assoc 1987; 114: 40-5.

5. Krogh H. Permanent tooth Mortality a Clinical study of cause of loss. J Am Dent Assoc 1958; 57: 670-5.

6. Cahen F, Frank R, Turlot C. Survery of the Reasons for dental Extractions in France. J Dent Res 1985; 64(8):1087-93.

7. Anderson DL, Thompson GW, Popovich F. Socioeconomic status, loss of teeth, and participation in a dental study. J Public Health Dent 1974; 34(2):106-12

8. Klein H. Tooth mortality and socioeconomic status-life tables for teeth. J Am Dent Assoc 1943; 39:80-95.

9. Malagon-Londoño G, Galan R, Ponton G. Administración Hospitalaria. Colombia: Panamericana; 1996.
10. Walton R. Endodoncia Principios y Practica, 2a ed. Madrid: Mc Graw Hill Interamericana; 1996.

11. Cohen S, Burns R. Vías de la pulpa. 7a ed. Madrid: Harcourt; 1999.

12. Trowbridge H. Inflammation. A review of the process. 5th ed. Chicago: Quintessence; 1997.

13. Seltzer S, Bender I. Dental Pulp Chicago: Quintessense; 2002.

14. Ingle J. Endodoncia. 3a ed. Mexico: Interamericana; 1987.

15. Ingle J. Endodoncia. 5a ed. Mexico: Mc Graw Hill Interamericana; 2002. p. 95-177.

16. Villena H. Terapia Pulpar. Lima : Universidad Peruana Cayetano Heredia; 2001.

17. Simon J, Walton R, Pashley D, Dowden W, Bakland L. Patosis Pulpar. 4a ed. McGraw-Hill Interamericana; 1996.

18. Cohen S, Burns R. Vías de la Pulpa. 7a ed. Madrid: Harcourt; 1999.

19. Harty FJ. A survey of endodontic procedures performed by practitioners in limited practice. Int Endod J 1992; 25(1):25-8.

20. Lasala A. Endodoncia. 3a ed. Madrid. Salvat; 1988.

21. Molven O. Tooth mortality and endodontic status of a selected population group. Observations before and after treatment. Acta Odontol Scand 1976; 34(2):107-16.

22. Barbakow FH, Cleaton-Jones P, Friedman D. An evaluation of 566 cases of root canal therapy in general dental practice. 1. Diagnostic criteria and treatment details. J Endod 1980; 6(2):456-60.

23. Serene TP, Spolsky VW. Frequency of endodontic therapy in a dental school setting. J Endod 1981; 7(8):385-7.

24. Gordon M, Tamse A, Metzger Z. Incidence and distribution of root canal treatments in the central Israeli military dental clinic: a 1-year survey. Int Endod J. 1988; 21(4):264-7.

25. Taintor JJ, Bierster RC, Fahid A. Profile of initial root canal treated teeth in private practice. J. Endod 1989; 15: 181.

26. Abeles Peraza GS. Motivación en
Odontología. Mexico:Salud Bucal; 1979.

27. Palmqvist $\mathrm{S}$, Soderfeldt B, Arnbjerg D. Dental conditions in a Swedish population aged 45-69 years. A questionnaire study. Acta Odontol Scand 1991; 49(6):377-84.

28. Harty FJ. A survey of endodontic procedures performed by practitioners in limited practice. Int Endod J 1992; 25(1):25-8.

29. Manning S. Profile of a specialist endodóntico practice. NZ Endod J 1992; 18:21-3.

30. Bermejo C. Demanda del servicio de emergencia estomatológica del sistema de servicios estomatológicos docente asistencial periodo enero 1991 a abril 1993. [Tesis de Bachiller] Lima: Universidad Peruana Cayetano Heredia; 1993.

31. Abbott PV. Analysis of a referralbased endodontic practice: Part 1. Demographic data and reasons for referral. J Endod 1994; 20(2):93-6.

32. Watanabe MG. Aspectos sócioeconômicos dos pacientes atendidos nas clínicas da faculdade de odontologia de Ribeirão Preto USP. Rev Odontol Univ São Paulo 1997; 11(2):147-51.

33. Brignardello C. Motivo de consulta de los pacientes que acudieron a la Clínica Estomatologica Central de la Universidad Peruana Cayetano Heredia durante los años 1991-1992. [Tesis de Bachiller] Lima: Universidad Peruana Cayetano Heredia; 1994.

34. Reátegui P. Frecuencia de retratamientos endodónticos según grupo dentario de pacientes que acudieron al servicio de post-grado de endodoncia de la Clínica Estomatológica Central Cayetano Heredia durante el período enero 1995 a enero 1997. [Tesis de Bachiller] Lima: Universidad Peruana Cayetano Heredia; 1998.

35. Márquez A. Frecuencia de motivos y lugar de referencia para tratamiento de conductos en la Clínica del Post Grado de Endodoncia de la Facultad de Estomatología de la UPCH entre Enero de 1995 y Enero de 1997. [Tesis de Bachiller] Lima: Universidad Peruana Cayetano Heredia; 1998. 
36. Valdivia R. Manifestaciones clínicas y hallazgos radiográficos más frecuentes en los diferentes diagnósticos pulpares de pacientes que acudieron al servicio de post-grado de endodoncia de la Clínica Estomatológica Central Cayetano Heredia durante el período enero 1995 enero 1997. [Tesis de Bachiller] Lima: Universidad Peruana Cayetano Heredia; 1998.

37. Salazar L. Determinación de la frecuencia de las piezas dentarias permanentes con mayor necesidad de tratamiento de conducto radicular en pacientes que acudieron al servicio de post-grado de endodoncia de la Clínica Estomatológica Central de la Universidad Peruana Cayetano Heredia, durante el período Enero 1995 a Enero 1997. [Tesis de Bachiller] Lima: Universidad Peruana Cayetano Heredia; 1999.

38. García R. Perfil de la demanda de atención en el Servicio de Endodoncia-Postgrado de la Universidad Peruana Cayetano Heredia. [Tesis de Maestría] Lima: Universidad Peruana Cayetano Heredia; 2000.
39. Flores M. Determinación del número de sesiones utilizadas en la realización de pulpectomías de piezas permanentes según el diagnóstico pulpar y periapical y el tipo de pieza dental en el área de postgrado de endodoncia de la Clínica Estomatológica Central de la Universidad Peruana Cayetano Heredia : período mayo 2000-abril 2001. [Tesis de Bachiller] Lima : Universidad Peruana Cayetano Heredia; 2001

40. Liu G. Factores asociados a la oferta y demanda que influyen en la utilización de servicios de la Clínica Estomatologica Central de la Universidad Peruana Cayetano Heredia durante los meses de diciembre 2000 a febrero 2001.[Tesis de Bachiller] Lima: Universidad Peruana Cayetano Heredia; 2001.

41. Chancafe J. Grado de preparación biomecánica en piezas dentarias uniradiculares con un conducto radicular de acuerdo al diagnóstico pulpar y periapical en pacientes tratados endodónticamente en el área de pre-grado de la Clínica Estomatológica Central de la Universidad
Peruana Cayetano Heredia, período Enero-Diciembre 2001. [Tesis de Bachiller] Lima: Universidad Peruana Cayetano Heredia; 2002.

42. Oyanguren S. Motivos de repetición de trabajos de laboratorio de producción entregados a operadores de la asignatura CIA V de la Clínica Estomatológica Central de la Universidad Peruana Cayetano Heredia en el año 2003. [Tesis de Bachiller] Lima: Universidad Peruana Cayetano Heredia; 2004.

43. Gulabivala K, Aung TH, Alavi A, Ng YL. Root and canal morphology of Burmese mandibular molars. Int Endod J 2001; 34(5):359-70.

44. Gutmann J. Problem solving in endodontics. 3rd ed. St. Louis: Mosby; 1997.

45. Lasala A. Endodoncia. 3 a ed. Madrid: Salvat editores; 1988.

46. Bennett CG, Kelln EE, Biddington WR. Age changes of the vascular pattern of the human dental pulp. Arch Oral Biol 1965; 10(6):995-8.

47. Lavelle CL. The effect of age on the pulp tissues of rat incisors. J Gerontol 1969; 24(2):155-6. 